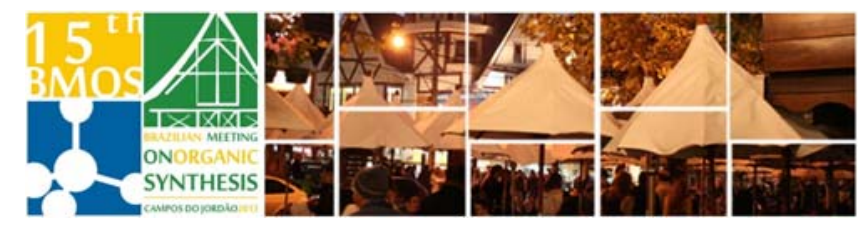

\title{
Towards the synthesis of 27-Deoxylyngbyabellin A analogues as potential chemotherapeutics.
}

\section{Laura Scarone, ${ }^{*}$ Leonardo Sandin and Gloria Serra.}

Cátedra de Química Farmacéutica, (DQO), Facultad de Química, UdelaR, Gral. Flores 2124, Montevideo,

$$
\text { CP 11800, Uruguay. }
$$

*e-mail: laurito@fq.edu.uy

Keywords: Lyngbyabellins, macrocycles, peptides.

\section{INTRODUCTION}

Natural products play an important role in drug development, particularly in anticancer, antibiotics and antiparasitics drugs. ${ }^{1}$ Marine cyanobacteria have been attracting increasing attention in drug discovery due to their structurally novel bioactive secondary metabolites. These natural products are predominantly modified peptides and depsipeptides, many of which happen to be cyclic. ${ }^{2}$

27-Deoxylyngbyabellin A (1, figure 1), which was isolated from the cyanobacteria Lyngbya bouillonii, showed moderate cytotoxicity toward cancer cell lines HT29 and HeLa. ${ }^{3}$

As part of our search for compounds as candidates for anticancer or antiparasitic drugs employing molecular simplification, ${ }^{4}$ we are interested in the preparation of depsipeptides analogues of type 2 . (figure 1)

Figure 1: 27-Deoxylyngbyabellin A and Depsipeptides analogues.

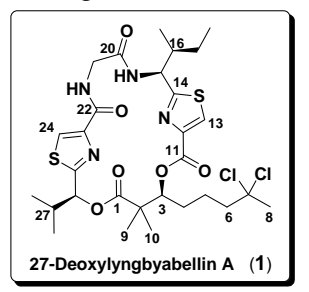

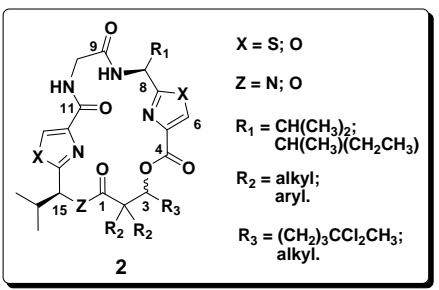

Herein, we presents the synthesis of fragment C4C15 of depsipeptide of type 2 with $\mathrm{X}=\mathrm{O}$ and $\mathrm{R}_{1}=$ $\mathrm{CH}\left(\mathrm{CH}_{3}\right)_{2}$.

\section{RESULTS AND DISCUSSION}

Building blocks synthesis:

The 1,3-oxa(thia)aza systems were obtained from $\beta$ hydroxyamide or thioamides using Xtal Fluor-E as cyclodehydrative reagent. $^{5}$ (Scheme 1 ) The amide from the L-Ser-OMe and $N$-Boc-L-Val was prepared employing HBTU as coupling reagent.

Protection of L-Ser hydroxyl group and then reaction with Lawesson's reagent rendered the desired thioamide.

Oxidation of oxazolines and thiazolines were performed with $\mathrm{BrCCl}_{3}$ and DBU.
Scheme 1: Building blocks construction

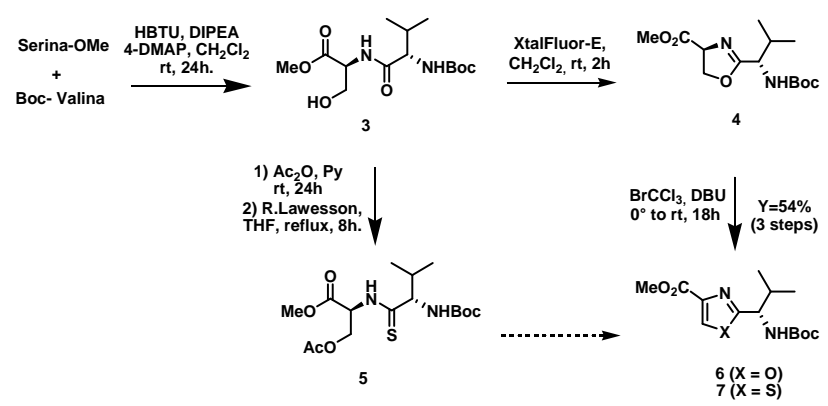

Fragments C4-C15:

A sequence of $N$-Boc cleavage and ester hydrolysis followed by the coupling of the adequate residue rendered fragment C4-C15 (9, Scheme 2) of depsipeptide of type 2.

Scheme 2: Fragment C4-C15 of depsipeptides type 2.

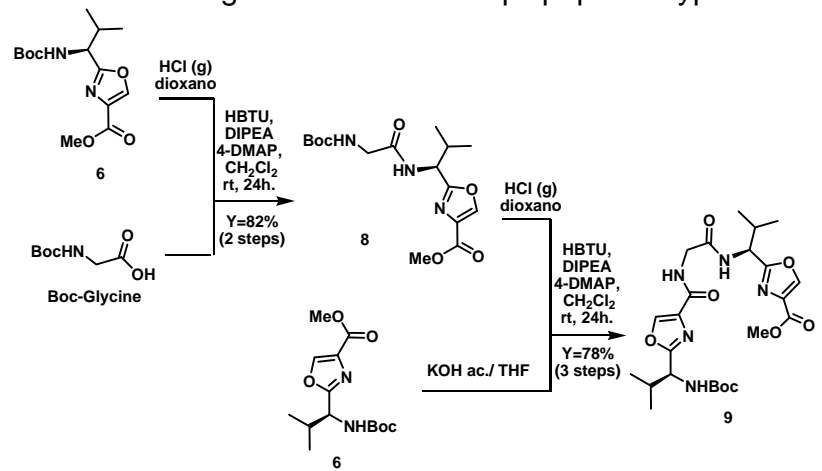

\section{CONCLUSION}

The employed methodology allowed us to prepare fragment C4-C15 of depsipeptide of type 2 with $\mathrm{X}=$ $\mathrm{O}$ and $\mathrm{R}_{1}=\mathrm{CH}\left(\mathrm{CH}_{3}\right)_{2}$ in good yields.

\section{ACKNOWLEDGEMENTS}

Facultad de Química, CSIC_Grupos, Pedeciba, Lab. RMN, MS.

\section{REFERENCES}

${ }^{1}$ Newman, D. J.; Cragg, G. M. J. Nat. Prod. 2012, 75, 311-335.

${ }^{2}$ Tan, L. T. Phytochemistry 2007, 68, 954-979.

${ }^{3}$ Matthew, S.; Salvador, L.A,; Schupp, P.J.; Paul, V.J.; Luesch, H. J. Nat. Prod. 2010, 73, 1544-1552.

${ }^{4}$ Peña, S.; Scarone, L.; Manta, E.; Stewart, L.; Yardley, V.; Croft, S.; Serra, G. Bioorg. Med. Chem. Lett. 2012, 22, 4994-4997.

${ }^{5}$ Pouliot, M.F.; Angers, L.; Hamel, J.D.; Paquin, J.F. Tetrahedron Lett. 2012, 53, 4121-4123. 